\title{
Stability Indicating Method Development and Validation of the Trandolapril in Human Plasma by LCMS/MS in Bulk and Tablet Dosage Form
}

\author{
Ganipisetty Lakshmi Aswini**1, D.Dachinamoorthy ${ }^{2}$, J. V. L. N. Seshagiri Rao ${ }^{3}$ \\ ${ }^{1}$ School of Pharmaceutical Sciences, Kakinada, Andhra Pradesh- 533003 \\ ${ }^{2}$ QIS College of Pharmacy, Ongole, Andhra Pradesh- 523272 \\ ${ }^{3}$ Srinivasa Rao College of Pharmacy, PM Palem, Vishakhapatnam, Andhra Pradesh- 530041
}

\begin{abstract}
A selective, sensitive and rapid HPLC-MS/MS method has been developed and validated for simultaneous quantification of trandolapril in human plasma using meloxicam as internal standard (IS). The analytes and IS were extracted from $200 \mu \mathrm{L}$ of plasma by liquid-liquid extraction technique. Chromatographic separation was achieved in 5.3 min and run time was 6.5min, on a Zorbax Eclipse $X D B$-Phenyl 3.5 $\mu, 7.5 \times 4.6 \mathrm{~mm}$ using a $0.1 \%$ formic acid and acetonitrile $(30 / 70 \mathrm{~V} / \mathrm{V})$ as mobile phase. The quantification of target compounds was performed in a positive electrospray ionization mode and multiple reaction monitoring (MRM). The proposed method was validated over the concentration ranges of $5.061-500.682 \mathrm{pg} / \mathrm{ml}$ for each analyte. The intra- and inter-day precision and accuracy results were acceptable as per FDA guidelines. Stability of compounds were established in a battery of stability studies, i.e. bench top, auto sampler, dry extract and long term storage stability as well as freeze-thaw cycles. The validated method has been successfully used to analyze human plasma samples for stability studies under various storage conditions.
\end{abstract}

Keywords: Trandolapril, meloxicam, HPLC-MS/MS, development, validation

\section{Introduction}

Trandolapril is a non-sulfhydryl prodrug that belongs to the angiotensin-converting enzyme (ACE) inhibitor class of medications. It is metabolized to its biologically active diacid form, Trandolaprilat, in the liver. Trandolaprilat inhibits ACE, the enzyme responsible for the conversion of angiotensin I (ATI) to angiotensin II (ATII). ATII regulates blood pressure and is a key component of the renin angiotensin aldosterone system (RAAS). Trandolapril may be used to treat mild to moderate hypertension, to improve survival following myocardial infarction in clinically stable patients with left ventricular dysfunction, as an adjunct treatment for congestive heart failure, and to slow the rate of progression of renal disease in hypertensive individuals with diabetes mellitus and microalbuminuria or over nephropathy.

Literature survey reveals that several methods have been reported for the quantitative determination of trandolapril individually in biological fluids. However, till date no LCMS/MS method has been reported in literature for the determination of trandolapril in human plasma. Hence, authors attempted to develop a simple and reproducible LCMS/MS method for simultaneous quantification of these three analytes which helps the researchers for therapeutic drug monitoring and pharmacokinetics. The aim of this work is, to develop a simple, selective and sensitive method, which employs liquid-liquid extraction technique for sample preparation and liquid chromatography with electrospray ionisation-tandem mass spectrometry for simultaneous quantitation of trandolapril in human plasma. The proposed method has significant advantages over earlier reported methods like Shorter run time, Wider linearity range with a more sensitivity and Simple reproducible extraction.

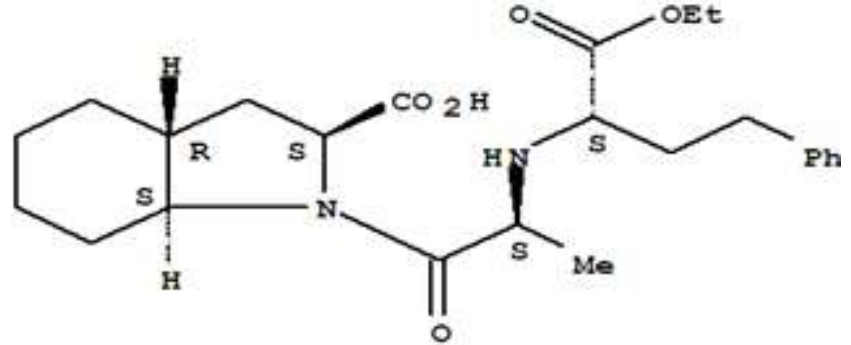

Figure 1: Chemical structures of trandolapril

\section{Materials and Method}

\subsection{Materials and reagents}

Reference standards of trandolapril (Figure. 1), internal standard (meloxicam) were purchased from Sigma-Aldrich (Hyderabad, India). HPLC grade methanol was procured from J.T Baker (Phillipsburg, USA). Milli-Q water (18.2 $\mathrm{m} \Omega$ and $\mathrm{TOC} \leq 50 \mathrm{ppb}$ ) from Milli-Q purification system, Millipore (Bangalore, India) was used throughout the study. Drug free human plasma was procured from King George Hospital (Visakhapatnam, India).

\subsection{Chromatographic and mass spectrometric conditions}

Equipment used is API 4000 LC-MS/MS coupled with UFLC model separation module. Mobile phase is $0.1 \%$ formic acid and acetonitrile $(30 / 70 \mathrm{~V} / \mathrm{V})$. The analytes and IS were separated by using Zorbax Eclipse XDB-Phenyl $3.5 \mu, 7.5 \times 4.6 \mathrm{~mm}$ column maintained at $40 \pm 20 \mathrm{C}$ with flow rate of $0.6 \mathrm{~mL} / \mathrm{min}$. The sample manager was maintained at $5^{\circ} \mathrm{C}$ and injection volume was $5 \mu \mathrm{L}$. The total chromatographic run time was $6.5 \mathrm{~min}$. The analytes and IS were detected using a Waters XEVO TQ mass spectrometer 


\section{International Journal of Science and Research (IJSR) \\ ISSN (Online): 2319-7064}

Index Copernicus Value (2016): 79.57 | Impact Factor (2015): 6.391

(Waters corporation, Milford, USA) equipped with Z spray source. The quantification of analytes and IS were achieved by operating the mass spectrometer in positive ion ESI with multiple reaction monitoring (MRM) mode. Nitrogen gas was used as both cone gas and desolvation gas with a flow rate of $50 \mathrm{~L} / \mathrm{Hr}$ and $800 \mathrm{~L} / \mathrm{Hr}$ respectively. The source dependent parameters capillary voltage, extractor voltage, source temperature and desolvation temperature were set at $3.50 \mathrm{KV}, 3 \mathrm{~V}, 150^{\circ} \mathrm{C}$ and $400^{\circ} \mathrm{C}$ respectively.

\subsection{Sample pretreatment}

A simple and rapid protein precipitation method was used for the preparation of plasma samples. Required number of plasma samples from the deep freezer was retreived and thawed them at room temperature or in a water bath maintained at room temperature and vortexed the tubes to mix. Transfer $200 \mu \mathrm{L}$ of plasma was transfered into prelabeled tubes. $100.0 \mu \mathrm{L}$ of extraction buffer was added to all the vials and vortex for about 5 seconds. $2 \mathrm{~mL}$ of tertiary butyl methyl ether (TBME) was added to the all vials and vortex for a period of $10 \mathrm{~min}$, interrupting the Vortexer for every 1 minute. All the vials were centrifuged at $4500 \mathrm{rpm}$, at $4^{\circ} \mathrm{C}$ for $5 \mathrm{~min}$. About $1.8 \mathrm{~mL}$ of supernatant was transferred into pre-labeled tubes and the supernatant solutions were evaporated to dryness under nitrogen at $40 \pm$ $2^{\circ} \mathrm{C}$. $500.0 \mu \mathrm{L}$ of reconstitution solution was added to all the tubes and vortexed for about 2 min.Appropriate volumes of the reconstituted solution were transferred into pre-labeled autosampler vials and $5 \mu \mathrm{L}$ was injected into LC-MS/MS.

\subsection{Preparation of calibration standards and quality control (QC) samples}

Stock solutions of trandolapril and IS were prepared in methanol at $1.0 \mathrm{mg} / \mathrm{ml}$ and $100 \mu \mathrm{g} / \mathrm{ml}$ respectively. The standard stock solution was prepared in a concentration range of $263.95-83400.00 \mathrm{ng} / \mathrm{ml}$, for trandolapril. The IS solution was similarly diluted to $2 \mu \mathrm{g} / \mathrm{ml}$ with methanol. Calibration standard samples of trandolapril were obtained by spiking $10 \mu \mathrm{l}$ of the appropriate working solutions to 100 $\mu$ blank plasma. Quality control (QC) samples at low, medium and high concentrations (713.37, 45036 and 83400 $\mathrm{ng} / \mathrm{ml}$ ) were prepared separately in the same fashion. All stock solutions, working solutions and quality control samples were stored at $-70{ }^{\circ} \mathrm{C}$ until used.

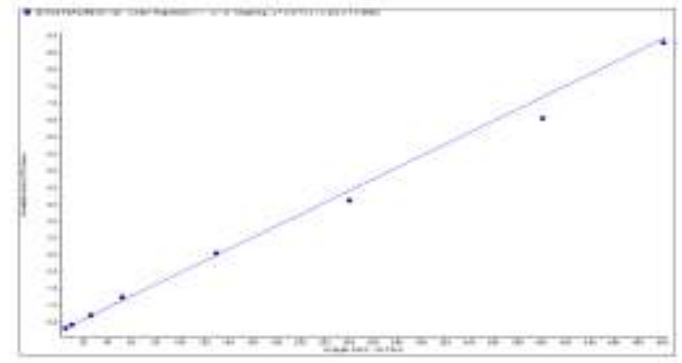

Figure 2: A Representative CalibrationCurve

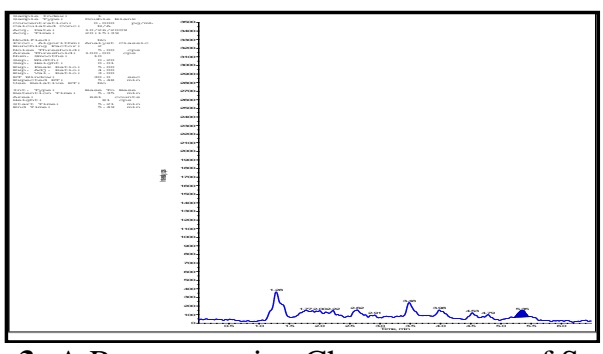

Figure 3: A Representative Chromatogram of Standard Blank

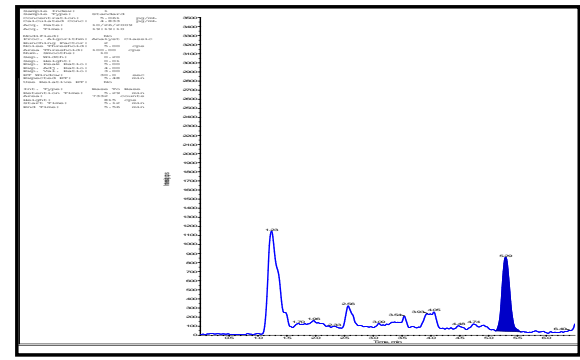

Figure 4: A Representative Chromatogram of LLOQ Standard

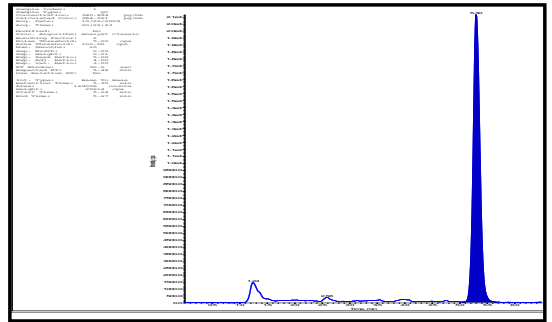

Figure 5: A Representative Chromatogram of HQC sample

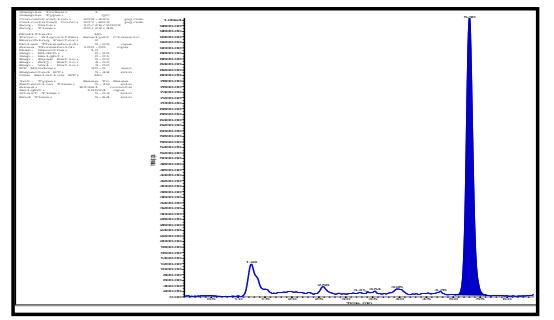

Figure 6: A Representative Chromatogram of MQC sample

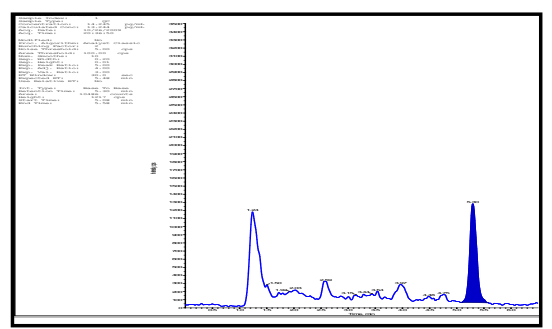

Figure 7: A Representative Chromatogram of LQC sample Figure 3-7 - MS/MS spectra of trandolapril and meloxicam (IS)

\section{Stability of Analytes}

\subsection{Stock Solution Stability}

3.1.1 Short Term Stock Solution Stability for Analyte and Internal Standard

Short term stock solution stability of the analyte was determined by storing SSHQC for07hrs $15 \mathrm{mins}$ at room temperature. The \% mean atability was found to be $99.97 \%$.

Volume 6 Issue 12, December 2017 


\section{International Journal of Science and Research (IJSR) \\ ISSN (Online): 2319-7064}

Index Copernicus Value (2016): 79.57 | Impact Factor (2015): 6.391

Short term stock solution stability of the IS was determined by storing SSHQC for 06hrs 20 mins at room temperature. The \% mean stability was found to be $99.92 \%$. The Stability was assessed by comparing with the freshly prepared solutions of both standard solution and internal standard. The acceptance limit was $90.00 \%-110.00 \%$.

Short Term Spiking Solution Stability for Analyte and Working Solution Stability for Internal Standard:

Short term stock solution stability of the analyte was determined by storing SSHQC and SSLQC for 07hrs 10 mins at room temperature. The $\%$ mean atability was found to be $100.20 \%$ and $99.49 \%$. Short term stock solution stability of the IS was determined by storing for $06 \mathrm{hrs}$ $30 \mathrm{mins}$ at room temperature. The \% mean stability was found to be $100.78 \%$.The Stability was assessed by comparing with the freshly prepared solutions of both standard solution and internal standard .The acceptance limit was $90.00 \%-110.00 \%$.

\subsubsection{Long Term Stock Solution Stability for Analyte and Internal Standard:}

Long term stock solution stability of the analyte was determined by storing SSHQC for 11days $16 \mathrm{hrs} 20 \mathrm{mins}$ at $5 \pm 3^{\circ} \mathrm{c}$. The $\%$ mean atability was found to be $99.79 \%$. Long term stock solution stability of the IS was determined by storing for 11 days $16 \mathrm{hrs} 20 \mathrm{mins}$ at $5 \pm 3^{\circ} \mathrm{c}$. The $\%$ mean stability was found to be $99.98 \%$. The Stability was assessed by comparing with the freshly prepared solutions of both standard solution and internal standard .The acceptance limit was $90.00 \%-110.00 \%$.

\section{Long Term Spiking Solution Stability for Analyte and Working Solution Stability for Internal Standard:}

Long term stock solution stability of the analyte was determined by storing SSHQC and SSLQC for11days $1 \mathrm{hr}$ at $5 \pm 3^{\circ} \mathrm{c}$. The $\%$ mean atability was found to be $100.18 \%$ and $101.52 \%$. For IS it was determined by storing for 11 days $1 \mathrm{hrs} 45 \mathrm{mins}$ at $5 \pm 3^{\circ} \mathrm{c}$. The $\%$ mean stability was found to be $99.86 \%$. The Stability was assessed by comparing with the freshly prepared solutions of both standard solution and internal standard. The acceptance limit was $90.00 \%-110.00$ $\%$.

\subsection{Stability of Analyte in Plasma:}

Stability studies in plasma were conducted in the various conditions using six replicates of LQC and HQC samples, as described below:

\subsubsection{Freeze Thaw Stability:}

Freeze thaw stability of the spiked quality control samples was determined after four freeze thaw cycles stored at -28 ${ }^{\circ} \mathrm{C} \pm 5{ }^{\circ} \mathrm{C}$. Stability was assessed by comparing them against the freshly spiked calibration standards and quality control samples. The \% mean stability for HQC and LQC was found to be $99.48 \%$ and $101.31 \%$ respectively, which is within the acceptance limit of $85.00 \%-115.00 \%$.

\subsubsection{Bench Top Stability:}

Bench top stability of the spiked quality control samples was determined for a period of 12 hours 24 minutes stored at room temperature. Stability was assessed by comparing them against the freshly spiked calibration standards and quality control samples. The \% mean stability for HQC and LQC was found to be $99.65 \%$ and $105.79 \%$ respectively, which is within the acceptance limit of $85.00 \%-115.00 \%$.

\subsubsection{Auto sampler Stability:}

Auto sampler stability of the processed quality control samples was determined for a period of 80 hours 05 minutes by storing them in auto sampler maintained at temperature 5 $\pm 3{ }^{\circ} \mathrm{C}$. Stability was assessed by comparing against the freshly spiked calibration standards and quality control samples. The \% mean stability for HQC and LQC was found to be $98.24 \%$ and $97.63 \%$ respectively, which is within the acceptance limit of $85.00 \%-115.00 \%$.

\subsubsection{Wet Extract Stability at Room Temperature:}

Wet extract stability of the spiked quality control samples was determined for a period of 24 hours 06 min by storing them at room temperature. Stability was assessed by comparing them against the freshly spiked calibration standards and quality control samples. The \% mean stability for HQC and LQC was found to be $98.28 \%$ and $106.30 \%$ respectively, which is within the acceptance limit of $85.00 \%$ $-115.00 \%$

3.2.5 Wet Extract Stability at Refrigerated Temperature: Wet extract stability of the spiked quality control samples wa888s determined for a period of 97 hours $55 \mathrm{~min}$ by storing them at $5 \pm 3{ }^{\circ} \mathrm{C}$. Stability was assessed by comparing them against the freshly spiked calibration standards and quality control samples. The \% mean stability for HQC and LQC was found to be $99.48 \%$ and $105.78 \%$ respectively, which is within the acceptance limit of $85.00 \%$ $-115.00 \%$.

\subsubsection{Dry Extract Stability}

Dry extract stability of the spiked quality control samples was determined for a period of 49 hours $55 \mathrm{~min}$ by storing them at $-28 \pm 5{ }^{\circ} \mathrm{C}$. Stability was assessed by comparing the stability samples against the freshly spiked calibration standards and quality control samples. The $\%$ mean stability for HQC and LQC was found to be $100.06 \%$ and $93.21 \%$ respectively, which is within the acceptance limit of $85.00 \%$ $-115.00 \%$

\subsubsection{Stability of Analyte in blood at Room Temperature} Stability of analyte in blood was determined at room temperature for a period of 03 hours and 05 minutes. Stability was assessed by comparing them against the freshly prepared samples in blood. The \% mean stability for HQC and LQC was found to be $101.00 \%$ and $98.43 \%$ respectively at room temperature, which is within the acceptance limit of $85.00 \%-115.00 \%$. The data of stability were summarized below in Table 1 .

\section{Volume 6 Issue 12, December 2017}




\section{International Journal of Science and Research (IJSR) \\ ISSN (Online): 2319-7064}

Index Copernicus Value (2016): 79.57 | Impact Factor (2015): 6.391

Table 1: Stability of trandolapril under various storage conditions

\begin{tabular}{|c|c|c|c|}
\hline & $\begin{array}{c}\text { Concentration } \\
(\mathrm{pg} / \mathrm{ml})\end{array}$ & $\begin{array}{c}\text { RSD } \\
(\%)\end{array}$ & $\begin{array}{c}\% \text { mean } \\
\text { stability }\end{array}$ \\
\hline Room temperature at 7 hrs & 380.89 & 1.38 & 100.20 \\
10 mins & 14.25 & 3.99 & 99.49 \\
\hline Storing at a period of 11 & 380.89 & 1.19 & 100.18 \\
days 1 hr & 14.25 & 1.88 & 101.52 \\
\hline Freeze thaw stability & 380.89 & 1.90 & 99.48 \\
stored at $-28^{\circ} \pm 5^{\circ} \mathrm{c}$ & 14.25 & 5.94 & 101.31 \\
\hline Bench top stability stored & 380.89 & 3.43 & 99.65 \\
at a period of 12hrs 24mins & 14.25 & 10.36 & 105.79 \\
\hline Auto sampler stability at & 380.89 & 2.70 & 98.24 \\
80 hrs 5mins & 14.25 & 5.78 & 97.63 \\
\hline Wet extract stabilityat 24 & 380.89 & 3.69 & 98.28 \\
hrs 6 mins & 14.25 & 5.68 & 106.30 \\
\hline Wet extract stability at & 380.89 & 1.07 & 99.48 \\
refrigerated temperature & 14.25 & 9.64 & 105.78 \\
\hline Dry extract stability at & 380.89 & 1.81 & 100.06 \\
49hrs 55mins & 14.25 & 11.98 & 93.21 \\
\hline Stability of analyte in & 380.89 & 8.24 & 101.0 \\
blood at room temperature & 14.25 & 0.57 & 98.43 \\
\hline
\end{tabular}

\subsection{Anticoagulant Effect}

Anticoagulant Effect was assessed by the spiked calibration standards and freshly prepared quality control samples in K3 EDTA human plasma. The \% mean stability for HQC and LQC was found to be $103.83 \%$ and $106.30 \%$ respectively, which is within the acceptance limit of $85.00 \%-115.00 \%$.

\section{Results and Discussion}

\subsection{Method development}

\subsubsection{Optimization of chromatographic and mass conditions}

In order to optimize MS condition, a standard solution of the trandolapril and IS were directly infused along with the mobile phase into the mass spectrometer withelectrospray ionization(ESI) asthe ionization source. Compared with the response observed in positive ion mode and negative mode, we discovered that trandolapril and IS had stronger signal intensity and lower background noise in positive ion mode. Therefore, detection was performed in positive ion mode in this study.

Chromatographic conditions played a critical role in achieving good chromatographic behavior. In this study, acetonitrile was chosen as the organic phase because of the advantage of higher responses and lower background noise by comparison with formic acid. Previous study indicates that low concentration of acid solution can facilitate the protonation and improve the intensity of basic compounds in the positive mode. Different concentrations of formic acid $(0.05 \%, 0.1 \%$ and $0.2 \%)$ were added to acetonitrile as different mobile phases. The shape of peak and intensities of trandolapril and IS were improved significantly when $0.1 \%$ formic acid was added in the mobile phase.

\subsubsection{Optimization of sample preparation}

Sample preparation is an important step for accurate and reliable LC-MS/MS assay. Several sample pre-treatment methods were investigated in this study, including protein precipitation and liquid-liquid extraction (LLE). However, the liquid- liquid extraction with tertiary butyl methyl ether showed limited extraction recovery efficiency, considering the polarity of the analytes. Thus, the protein precipitation method which was simpler and less time consuming was finally used. Different precipitation organic solvents were evaluated. Results showed that a precipitation with acetonitrile provided a higher recovery and perfect peak shape for both trandolapril and IS.

\subsubsection{Selection of internal standard}

According to the US Food and Drug Administration (FDA) guidance, any IS used in biological analysis should be a structurally similar analog of the analytes or a stable labeled compound. In this study ramipril and meloxicam were tested. Where ramipril has given unsatisfactory response. Therefore, meloxicam was chosen as the IS, because of the high ionization response in ESI mass spectrometry and a similar chromatographic retention time.

\subsection{Method validation}

\subsubsection{Specificity}

The detection of trandolapril and IS by MRM mode was highly selective with no interference from the endogenous substances. Fig.5 represents typical chromatograms of blank plasma, blank plasma spiked with trandolapril at LLOQ and IS. Typical retention times for trandolapril and IS were 5.3 min and $1.24 \mathrm{~min}$, respectively.

\subsubsection{Linearity and LLOQ}

The calibration curves showed good linearity over the concentration range of $0.5-5000 \mathrm{ng} / \mathrm{ml}$ in human plasma. A typical equation of the calibration curves was $\mathrm{y}=0.0173 \mathrm{x}+$ 0.253 ( $r=0.9969)$, where $y$ was the peak area ratio of trandolapril to IS and $\mathrm{x}$ was the concentration of trandolapril. The LLOQ for trandolapril in human plasma was $5.061 \mathrm{pg} / \mathrm{ml}$.

\subsubsection{Recovery and matrix effect}

The extraction recovery was in the range of $85.0-115.0 \%$ for trandolapril, which could meet the requirements of analysis. Results of matrix effects indicated that no significant matrix effects were observed for the analytes.

\subsubsection{Precision and accuracy}

The results of intra- and inter-day precision and accuracy at three concentration levels of the QC samples (380.89, $209.49,14.24$ and $5.27 \mathrm{pg} / \mathrm{ml}$ ) were summarized in Table 2. The intra- and inter-day RSD were measured to be below $13.21 \%$ and $15.64 \%$

The results demonstrated that both the intra- and inter-day assay values were all within the acceptable range.

\subsubsection{Stability}

Trandolapril had no effect on stability in autosampler ( $80 \mathrm{hrs}$ 5 mins) through repeated four freeze-thaw cycles and under the frozen condition at $-28 \pm 5^{\circ} \mathrm{C}$. 


\section{International Journal of Science and Research (IJSR) \\ ISSN (Online): 2319-7064}

Index Copernicus Value (2016): 79.57 | Impact Factor (2015): 6.391

Table 2: Precision and accuracy

\begin{tabular}{|l|c|c|c|c|c|}
\hline & Pg/ml & Intra day & Inter day & Accuracy & \%Recovery \\
\hline HQC & 380.89 & 3.27 & 2.37 & 101.56 & 82.87 \\
\hline MQC & 209.49 & 4.21 & 3.9 & 103.96 & 89.32 \\
\hline LQC & 14.24 & 8.92 & 13.02 & 94.67 & 87.15 \\
\hline LLQC & 5.27 & 13.21 & 15.64 & 107.07 & 86.44 \\
\hline
\end{tabular}

\section{Conclusion}

\subsection{Conclusion on the Chromatographic Method}

Based on the experiments done during the course of validation, it can be concluded that the intended method is validated for the estimation of Trandolapril in human plasma over the concentration range of 5.061-500.682 $\mathrm{pg} / \mathrm{ml}$. The precision and mean accuracy are within the acceptable limits. Consistent recoveries are observed for LQC, MQC and HQC. The method is specific enough in the presence of K2EDTA anticoagulant. The method is precise and accurate enough to dilute the samples, if necessary.

This method can be used for quantification of Trandolapril in human plasma for Bioequivalence studies.

\subsection{Conclusion on Stability of Analyte}

Based on the stability experiments carried out during the course of validation, it can be concluded that the intended analyte is stable in all the performed experiments and the stability experiments performed are within the acceptable limits.

\section{Acknowledgements}

The authors gratefully acknowledge Chandra Laboratories, India for providing necessary facilities to carry out this work

\section{References}

[1] Dubey S K. et.al, 2010. A high performance liquid chromatographic method for Quantification of Trandolapril using uv spectrometric detection. International journal of Biomedical Research, 1(3),133140.

[2] A.S.K.Sankar, M.Alekhya, Suraj Sythana, Dr.V.Ravichandiran. Analytical method development and validation of trandolapril in tablets by rp-hplc. International journal of frontiers science and technology. April-june-2013,1(2),119-127.

[3] Leena A. A Hawash, Ashok K. Shakya and Maher L. Saleem. Stability-Indicating HPLC Determination of Trandolapril in Bulk Drug and Pharmaceutical Dosage Forms. International journal of analytical chemistry. 2015.

[4] A. Gumieniczek \& H. Hopkala. Development and validation of a liquid chromatographic method for the determination of trandolapril and verapamil in capsules. 2007; 393-400.

[5] A.l rao, r.v bhaskara.,Development and validation of RP-HPLC method for the estimation of trandolapril and verapamil in combined tablet dosage form. 2012;49(5):61.
[6] Raju V. Bhaskara1, Rao A. Lakshmana, A Simple and Sensitive RP-HPLC Method for Estimation of Trandolapril in Bulk and Tablet Dosage Forms,Asian journal of research in chemistry,2011,4(9),1423.

[7] GanipisettyLakshmi Aswini, D. Dachinamoorthy, J. V. L. N.Seshagiri rao.Simultaneous estimation of Trandolapril and verapamil Hydrochloride in bulk and commercial products using a validated reverse phase high performance liquid chromatographic technique. international journal for pharmaceutical research scholars,2015;vol:4(4),1-9.

[8] Sahu, Kapendra; Karthikeyan, Chandrabose; S. H. N. Moorthy, Narayana; Trivedi, Piyush. A Validated UPLC Method Used for the Determination of Trandolapril and its Degradation Products as per ICH Guidelines, Current Pharmaceutical Analysis, 2011; 7(3):182-8.

[9] N. Sreekanth, Bahlul Z. Awen and Ch. Babu Rao . hptlc method development and validation of trandolapril in bulk andpharmaceutical dosage J Adv Pharm Technol Res. 2010 Apr-Jun; 1(2):172-179.

[10] Kowalczuk, Dorota.2005.Simultaneous highperformance thin-layer chromatography densitometric assay of Trandolapril and verapamil in the combination preparation. Journal of AOAC International, 88(5), 1525-29.

[11] Constantinos Pistos, MariaKoutsopoulou, IrenePanderi Liquid chromatographic tandem mass spectrometric determination of trandolapril in human plasma, Analytica Chimica Acta ,2005;540(2):375-82.

[12] Ramakrishna V. S. Nirogi, Vishwottam N. Kandikere, Wishu Shrivastava, Koteshwara Mudigonda, Quantification of trandolapril and its metabolite trandolaprilat in human plasma by liquid chromatography/tandem mass spectrometry using solidphase extraction. 2006;20(24),3709-16. 